\title{
Prediction of incremental spacing in press bend forming of the aluminum alloy integral panel
}

\author{
Cailing $\mathrm{Li}^{1, \mathrm{a}^{*}}$, Chao Cui ${ }^{1, \mathrm{~b}}$, Xiaoyu Shen ${ }^{1, \mathrm{c}}$, Zhe Wang ${ }^{1}$, YuLiang \\ Zhang ${ }^{1}$, Liming $\mathrm{Hu}^{1}$, Xiaoming Lai ${ }^{1}$, ZhanJiao $\mathrm{Gao}^{1}$, Weidong $\mathrm{Li}^{2}$ \\ ${ }^{1}$ Beijing spacecrafts, Beijing,China \\ ${ }^{2}$ Beijing University of Aeronautics and Astronautics, Beijing China \\ a lindali6@126.com, ${ }^{\mathrm{b}}$ cuichao20012001@163.com, ${ }^{\mathrm{c}}$ xiaoyu.shen@163.com
}

Key words: Aluminum alloy; Integral panel; incremental spacing

Abstract. In order to predict incremental spacing in press bend forming of Aluminum alloy integral panel, the typical integral panel test piece was taken as an example. By using numerical simulation method, this paper analyzed the effects of different incremental spacing on integral panel forming quality which included stress and strain distribution, curvature continuity, and surface profile. According to simulation results, experimental verification was implemented. The verification results show that the numerical simulation method to predict the incremental spacing is effective.

\section{Introduction}

Integral Panel[1] which has many advantages ,such as significant weight loss, high structural efficiency, integrity, build good coordination and so on , has a wide application in the field of aerospace. The technology of the Integral Panel forming has been a hot issue in the aerospace industry. Press bend forming is one of the main methods of integral panel forming. When it is forming, the expanding sheet is making plastic deformation between the upper and lower dies under normal temperature conditions to obtain a certain shape part.

Since Integral Panel is mostly large structure, generally, its forming method uses incremental press bend forming. Incremental press bend forming is deforming from local deformation accumulating into a whole deformation. It can effectively control the uniformity and reduce rib buckling and cracking, deformation damage, and production costs. It is widely used nowadays.

Using the incremental bend forming, incremental spacing between two adjacent steps is an important parameter which affects shape and quality of the Integral Panel. To shorten the development cycle, save costs, reduce the number of bending test quantity and get a reasonable incremental spacing, numerical simulation analysis is applied to the Integral Panel bending forming process. 


\section{Simulation analysis}

Simulation Model .This paper takes the typical integral panel test piece as an example, the shape and size of the test piece is shown in Figure 1.Characteristics of the test piece are high and narrow bars, uneven thickness skin. They increase the complexity and difficulty of the forming.
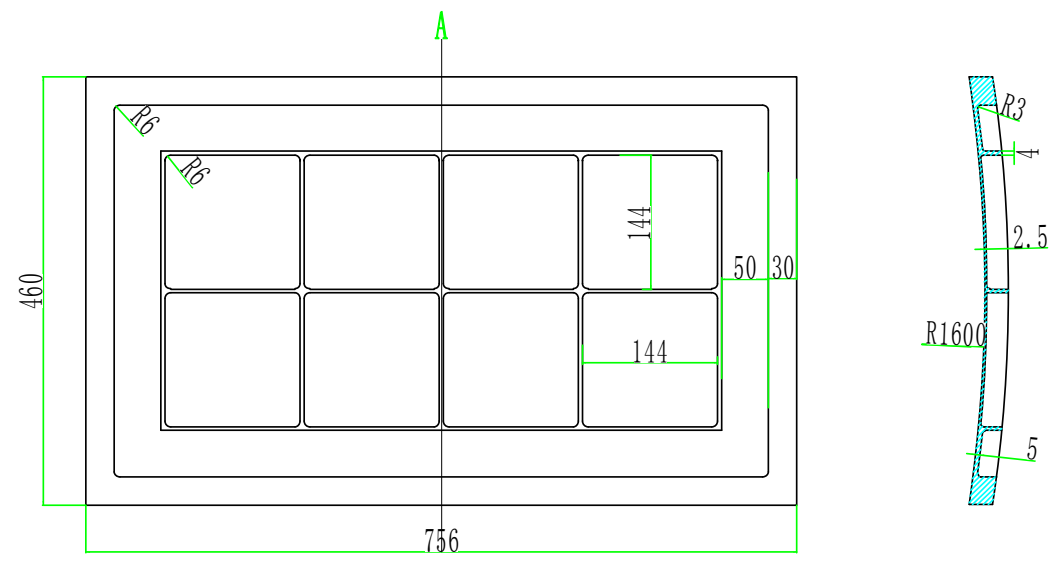

Figure 1 test piece

According to the Figure 1, the finite element model for Integral Panel incremental bending forming in FEM software ABAQUS is built, as shown in Figure 2.

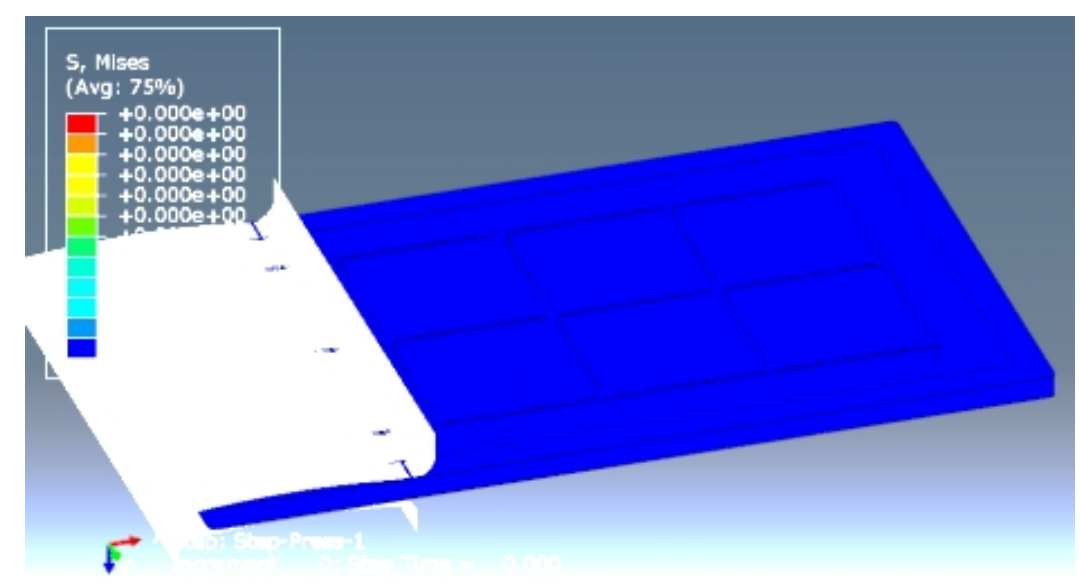

Figure2 element model

The process of Integral Panel incremental bending forming is Multi-step forming which is shown in Figure 3.In the process, expanding sheet is cycling the steps" pressed -unloaded-moved to next step", until the test piece is successfully formed. 


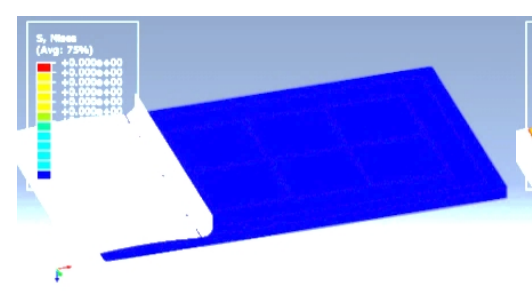

(1) initial

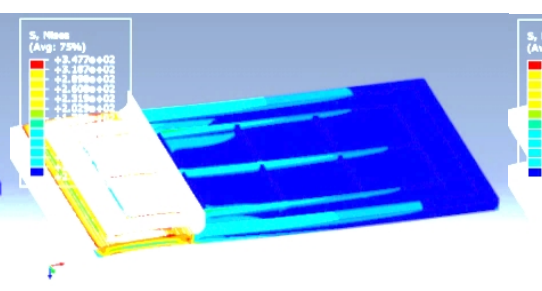

(2) first pressed

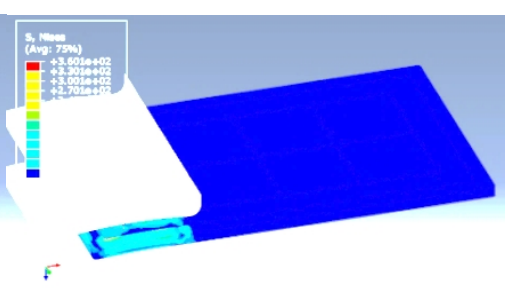

(3) first unloaded

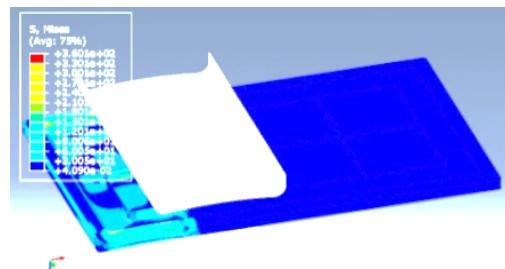

(4) moved to the second step

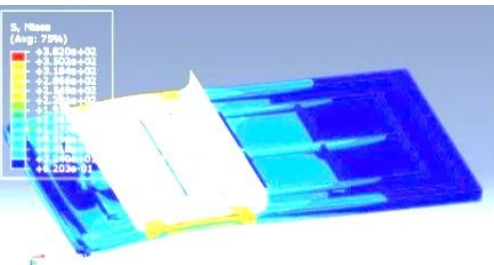

(5) second pressed

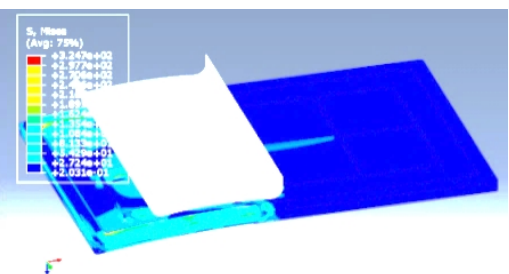

(6) second unloaded

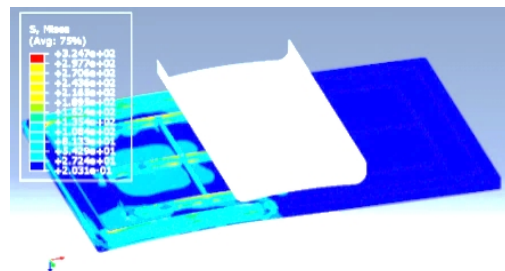

(7) moved to the third step

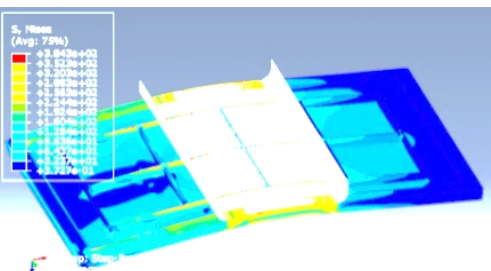

(8) third pressed

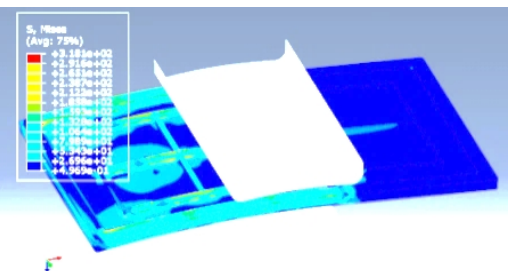

(9) third unloaded

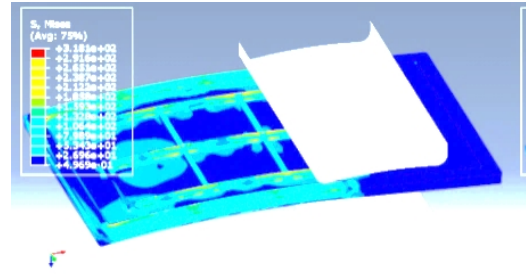

(10) moved to the forth step

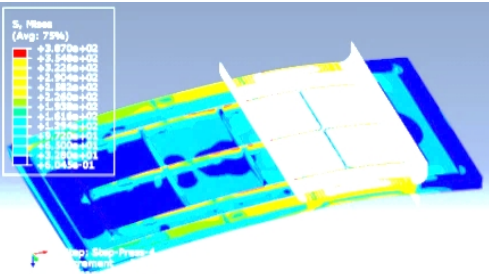

(11) forth pressed

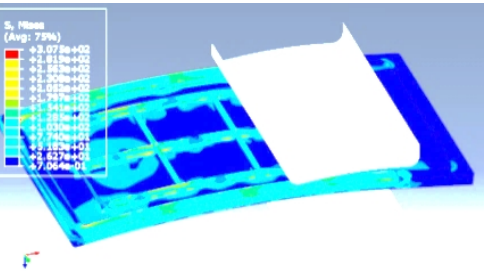

(12) forth unloaded

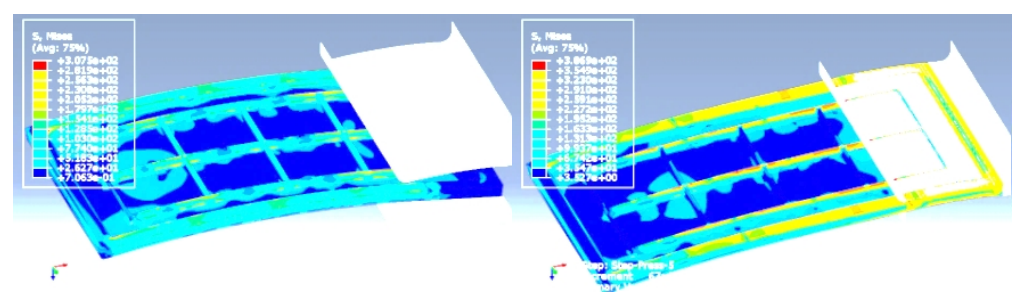

(13) moved to the fifth step

(14) fifth pressed

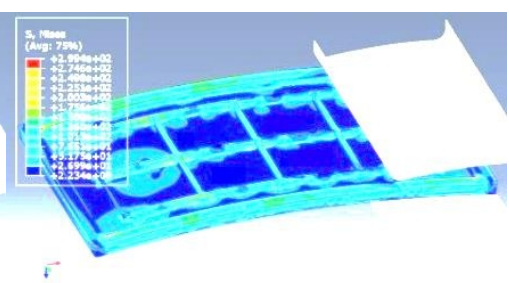

(15) fifth unloaded

Figure 3 the process of Integral Panel incremental bending forming

The material of the test piece is 5B70 which has low elongation and high strength, and it is one of the difficult-to-deform materials. In order to ensure the accuracy of the simulation, the plastic constitutive model parameters are obtained by using tensile tests. The elastoplastic stress-strain relationship of the material meets the constitutive model proposed by Wagoner [2], namely

$$
|\sigma|= \begin{cases}E|\varepsilon| & |\sigma| \leq \sigma_{s} \\ \sigma_{s}+K\left|\varepsilon_{P}\right|^{n} & |\sigma|>\sigma_{s}\end{cases}
$$

$\varepsilon$ is the true strain during elastic deformation, $\varepsilon_{p}$ is the plastic strain, $\sigma_{s}$ is yield strength, $\mathrm{n}$ is 
the material hardening exponent, $\mathrm{K}$ is the strength coefficient of the material, $\sigma$ is the true stress, and $\mathrm{E}$ is the elastic modulus of the material. Mises isotropic model is applied to the calculate yield model, and the true stress-strain curve of the material is shown in Figure 4.

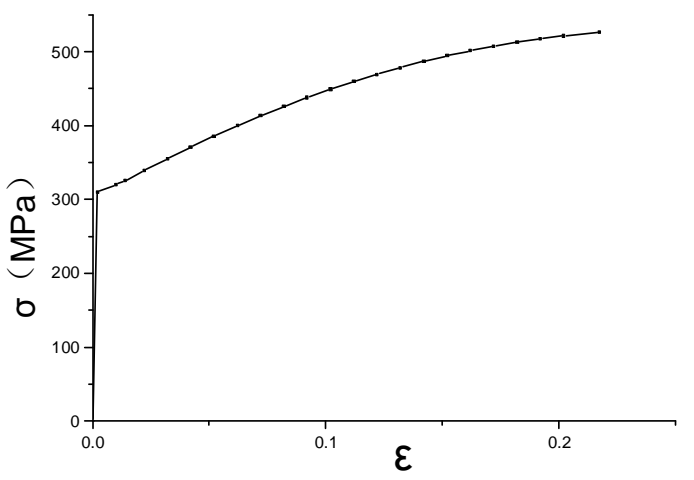

Figure 4 Materials true stress-strain curve

Simulation discussion. Against width of $200 \mathrm{~mm}$ incremental die, this paper uses $150 \mathrm{~mm}$, $200 \mathrm{~mm}$ and $300 \mathrm{~mm}$ incremental spacing respectively to simulate analysis. Analyze stress, strain, curvature continuity and outline degree of the test piece, in order to compare different incremental spacing which effects on the incremental bend forming.

Through the finite element analysis, Mises stress distribution is shown in Figure 5. You can see little difference between stress peaks under different incremental spacing. From the point of stress distribution, the best incremental spacing is $150 \mathrm{~mm}$.

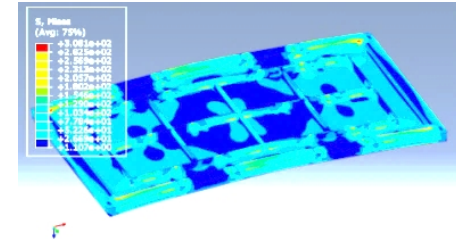

(a) spacing $300 \mathrm{~mm}$

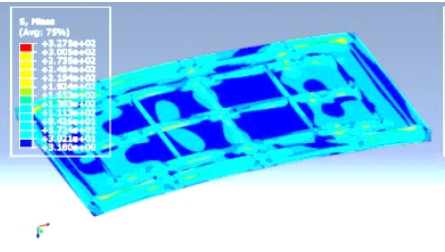

(b) spacing $00 \mathrm{~mm}$

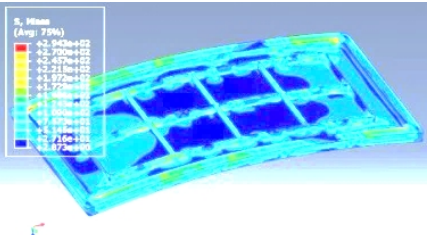

(c) spacing $150 \mathrm{~mm}$

Figure 5 Mises stress distribution

Maximum plastic principal strain distribution of the panel is shown in Figure 6. It can be seen little difference from maximum plastic strain main peak under different incremental spacing. From the point of strain distribution, the best incremental spacing is $150 \mathrm{~mm}$.

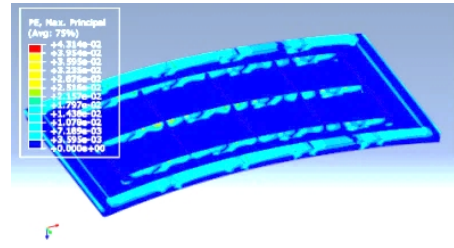

(a) spacing $300 \mathrm{~mm}$

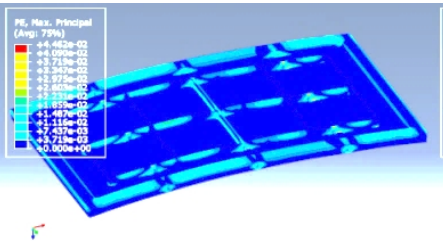

(b) spacing $200 \mathrm{~mm}$

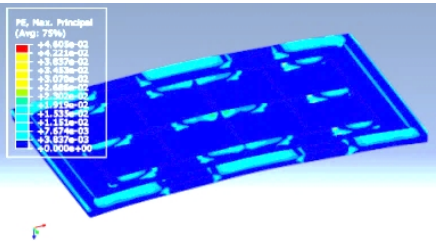

(c) spacing $150 \mathrm{~mm}$

Figure 6Maximum plastic principal strain distributions

Respectively, continuous curvature distribution and sectional curvature distribution of the panel are shown in Figure 7 and Figure 8.Obviously, the forming effect is significantly different under different incremental spacing. With spacing reducing, forming effect is getting better. When the 
spacing is $150 \mathrm{~mm}$, the panel meets the design requirements after forming.

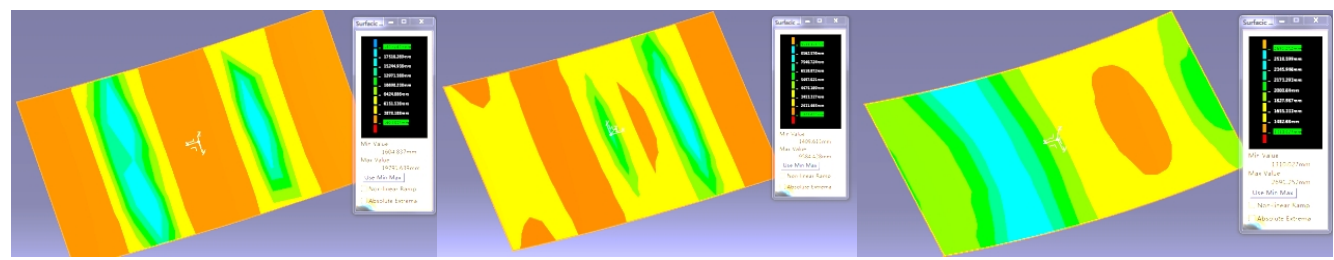

(a) spacing $300 \mathrm{~mm}$

(b) spacing $200 \mathrm{~mm}$

(c) spacing $150 \mathrm{~mm}$

Figure 7 Continuous curvature distributions

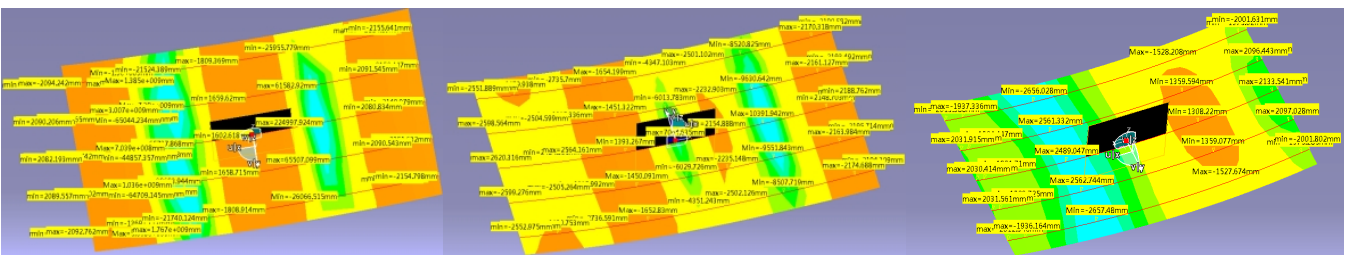

(a) spacing $300 \mathrm{~mm}$

(b) spacing $200 \mathrm{~mm}$

(c) spacing $150 \mathrm{~mm}$

Figure8 Sectional curvature distribution

Compared with the target surface profile, the simulation results are shown in Figure 9.The surface profile is getting better as the spacing decreases, when the spacing is $150 \mathrm{~mm}$, the surface profile meets design requirements.

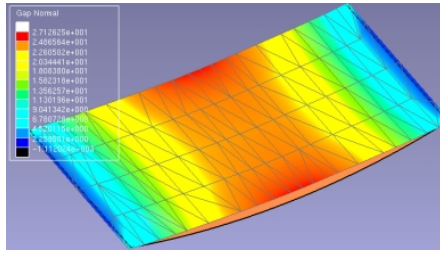

(a) spacing $300 \mathrm{~mm}$

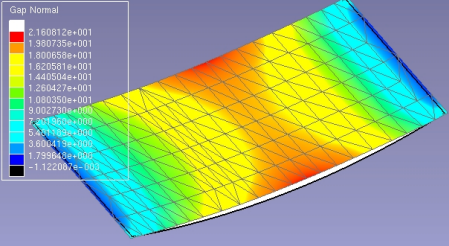

(b) spacing $200 \mathrm{~mm}$

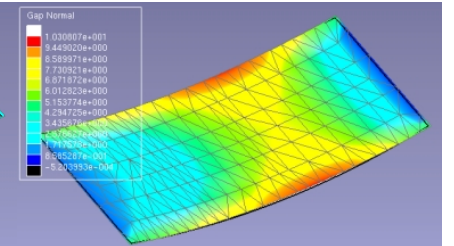

(c) spacing $150 \mathrm{~mm}$

Figur9 simulation results compared with the target surface profile

\section{Experiment Verification}

According to finite element numerical simulation results, the target integral panel and a similar panel were bent by using incremental spacing $150 \mathrm{~mm}$.

As shown in Figure 11, the quality of the integral panel test piece is good. The panel has uniform curvature and continuous arc. The shape of stiffeners and flanges is smooth. It has no cracking, distortion, or wrinkling. The straightness is high along the bus direction. The radius and contour of the design can be controlled within the tolerance range. All of them meet the requirements of the design dimensional accuracy. Experiments show that the method to predict the incremental spacing is effective and it has high reliability. This method can instruct the research and development of the product. 


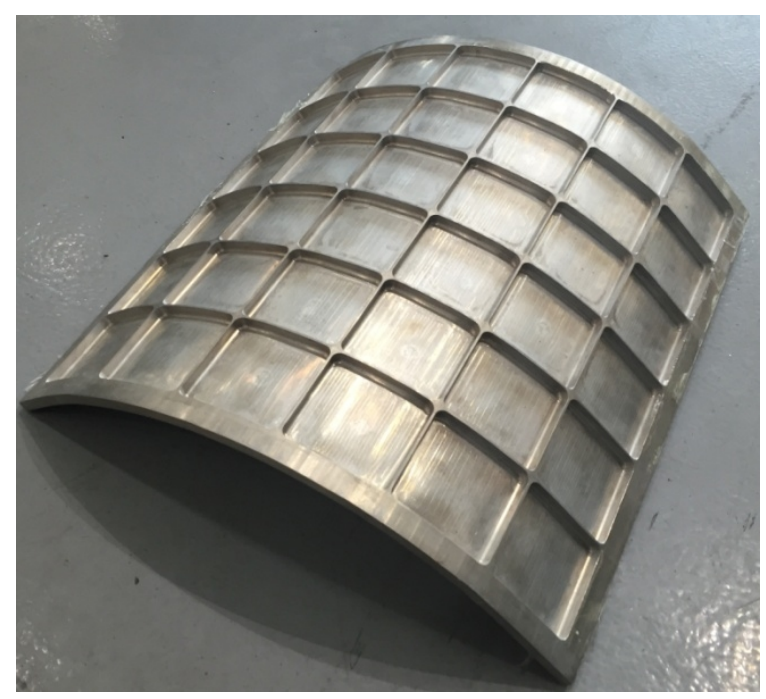

Figure 10 test piece

\section{Conclusions}

Based on the characteristics of the integral panel, the effects of the incremental spacing on the incremental bending forming quality and accuracy by simulation and experiment are analyzed in this paper. The conclusions are as follows.

(1)Forming quality is getting better as the incremental spacing decreases, when the incremental spacing is $150 \mathrm{~mm}$, a variety of precision meets the design requirements.

(2)The method how to predict the reasonable incremental spacing by simulation is practicable.

(3)The optimization result can be used as a basis for instructing the research and development of the follow-up products. It has a wide range of application prospects.

\section{References}

[1]Andrew Levers. Finite element analysis of creep age forming[C]. ABAQUSUSERS CONFERENCE, 1998: 461-474

[2] GangL, Wagoner R H. Spring unloaded analysis with a modified non-linear hardening model[J]. SAE2000, 2000-01-0410: 2000. 